\title{
Persistence of Vibrational Collectivity in Nuclear Level Densities
}

\author{
H. Nakada ${ }^{1, a}$, E. Matsuyama ${ }^{1}$, and C. Özen ${ }^{2}$ \\ ${ }^{1}$ Department of Physics, Graduate School of Science, Chiba University, Inage, Chiba 263-8522, Japan \\ ${ }^{2}$ Faculty of Engineering and Natural Sciences, Kadir Has University, Cibali 34083, Istanbul, Turkey
}

\begin{abstract}
By applying the particle-number projection to the finite-temperature BCS theory to the state densities in the rare-earth nuclei and comparing its results to the SMMC ones, we investigate effects of the particle-number conservation on the collective enhancement factor for the state densities. Once we restore the particle-number conservation, the rapid decay of vibrational enhancement disappears. This suggests that the vibrational collectivity may survive up to higher energy and the relevant enhancement factor is insensitive to the excitation energy.
\end{abstract}

\section{Introduction}

Nuclear level densities are one of the key quantities in understanding compound nuclear reactions. Widely used conventional approaches to nuclear level densities are based on the mean-field (MF) theories including the Fermi-gas model [1], which are suitable for global description covering almost the whole region of the nuclear chart. However, it has been known that collective excitations are missed in the MF approaches, and so-called collective enhancement factor (CEF) has been phenomenologically introduced as complements. Although the CEF is considered to represent the vibrational and the rotational collective degrees of freedom, microscopic understanding of the CEF has not been sufficient.

The shell model gives us a microscopic framework suitable for studying nuclear level densities, because it is a full quantum theory that can describe both collective and non-collective states in the model space. Although the model space required for reasonable description of level densities is much larger than tractable in the conventional diagonalization methods, the shell model Monte Carlo (SMMC) methods [2] enables us to overcome this difficulty. Indeed the SMMC methods have successfully been applied to the state densities of medium- to heavy-mass nuclei [3-9]. With respect to the collective excitations, the SMMC methods have successfully described crossover from the vibrational to the rotational collectivity in the rare-earth nuclei with a single class of Hamiltonians [10, 11]. On this basis, the energy-dependence of the CEF has been investigated from microscopic standpoint, by comparing the SMMC densities to the corresponding finite-temperature (FT) Hartree-Fock-Bogolyubov (HFB) densities [10]. It has been found that the decays of the CEF seem to correlate to the phase transitions in HFB; the decay of the vibrational enhancement to the pairing transition and that of the rotational enhancement to the shape transition. However, in MF approaches such as the HFB, certain symmetries are broken due to phase transitions, although those symmetries should hold in actual nuclei. Therefore, restoration of the broken symmetry is important to investigate genuine effects of the collectivity.

ae-mail: nakada@faculty.chiba-u.jp 
We here focus on the breaking and restoration of the global gauge symmetry, which is connected to the particle-number conservation. The FT-MF approaches are formulated in the grand-canonical ensembles, in which the thermal average is taken in the entire Fock space. In contrast, the nuclear level densities are argued for a specific nucleus with fixed proton and neutron numbers. Therefore the canonical ensembles are more appropriate for describing level densities, as adopted in the SMMC calculations. Reduction from the grand-canonical ensembles to the canonical ensembles in the FTMF theories is inspected recently [12]. However, in finite quantum systems such as nuclei, the phase transitions observed in the MF regime are washed out in truth [3], and full restoration is often desired to investigate influence of the existing symmetry. In the present study, we apply the particle-number projection [13-15] to the FT Bardeen-Cooper-Schrieffer (BCS) theory, and investigate effects of the symmetry restoration.

\section{Particle-number projection in FT-BCS theory}

\subsection{Thermodynamical quantities}

For canonical ensembles with a given Hamiltonian $H$, the statistical density operator at temperature $T=1 / \beta$ is defined by

$$
w^{(\mathrm{C})}=\frac{e^{-\beta H}}{\operatorname{Tr}_{\mathrm{C}}\left(e^{-\beta H}\right)},
$$

by which the thermal expectation value of an observable $O$ is calculated as

$$
\langle O\rangle_{\beta}^{(\mathrm{C})}=\operatorname{Tr}_{\mathrm{C}}\left(O w^{(\mathrm{C})}\right) .
$$

Here $\operatorname{Tr}_{\mathrm{C}}$ denotes the canonical trace.

The statistical density operator for grand-canonical ensembles is defined by

$$
w^{(\mathrm{GC})}=\frac{e^{-\beta\left(H-\sum_{\tau} \mu_{\tau} N_{\tau}\right)}}{\operatorname{Tr}_{\mathrm{GC}}\left(e^{-\beta\left(H-\sum_{\tau} \mu_{\tau} N_{\tau}\right)}\right)},
$$

where $\operatorname{Tr}_{\mathrm{GC}}$ denotes the grand-canonical trace, $N_{\tau}$ is the number operator and $\mu_{\tau}$ represents the chemical potential, with $\tau(=p, n)$ distinguishing protons and neutrons. The thermal expectation value of an observable is obtained by

$$
\langle O\rangle_{\beta}^{(\mathrm{GC})}=\operatorname{Tr}_{\mathrm{GC}}\left(O w^{(\mathrm{GC})}\right) .
$$

In the FT-MF theories, the Hamiltonian in $w^{(\mathrm{GC})}$ is approximated by the MF Hamiltonian $H_{0}$, giving

$$
w_{\mathrm{MF}}^{(\mathrm{GC})}=\frac{e^{-\beta\left(H_{0}-\sum_{\tau} \mu_{\tau} N_{\tau}\right)}}{\operatorname{Tr}_{\mathrm{GC}}\left(e^{-\beta\left(H_{0}-\sum_{\tau} \mu_{\tau} N_{\tau}\right)}\right)},
$$

The MF Hamiltonian has the form

$$
H_{0}=E_{0}+\sum_{k} \varepsilon_{k} \alpha_{k}^{\dagger} \alpha_{k},
$$

where $E_{0}$ is a constant, $k$ stands for a quasiparticle (q.p.) state and $\alpha_{k}^{\dagger}\left(\alpha_{k}\right)$ the corresponding creation (annihilation) operator. The thermal expectation value in the FT-MF approximations is

$$
\langle O\rangle_{\beta, \mathrm{MF}}^{(\mathrm{GC})}=\operatorname{Tr}_{\mathrm{GC}}\left(O w_{\mathrm{MF}}^{(\mathrm{GC})}\right) .
$$


In any case above, the energy expectation value is calculated by $E(\beta)=\langle H\rangle_{\beta}=\operatorname{Tr}(H w)$, and the heat capacity by $C=d E / d T=-\beta^{-2} d E / d \beta$. The entropy can be represented as

$$
S=-\operatorname{Tr}(w \ln w)
$$

Within the FT-MF framework, this is reduced to

$$
S_{\mathrm{MF}}^{(\mathrm{GC})}=-\sum_{k}\left[f_{k} \ln f_{k}+\left(1-f_{k}\right) \ln \left(1-f_{k}\right)\right],
$$

where (assuming the q.p. state $k$ belongs to the particle type $\tau$ )

$$
f_{k}=\left\langle\alpha_{k}^{\dagger} \alpha_{k}\right\rangle_{\beta, \mathrm{MF}}^{(\mathrm{GC})}=\frac{1}{e^{\beta\left(\varepsilon_{k}-\mu_{\tau}\right)}+1} .
$$

At thermal equilibrium, the free energy $F=E-T S$ takes minimum for a given temperature $T$ in the canonical ensembles, while the grand potential $\Omega=F-\sum_{\tau} \mu_{\tau} N_{\tau}$ does in the grand-canonical ensembles. $H_{0}$ in the FT-MF theories is determined so as to minimize $\Omega_{\mathrm{MF}}^{(\mathrm{GC})}$.

\subsection{Particle-number projection}

In Ref. [12] an approximate method of reducing the grand-canonical ensemble to the canonical ensemble is proposed and applied to the FT-MF theories. To restore the particle-number conservation exactly, it is desired to apply the projection operator on the proton and neutron numbers $\left(n_{p}, n_{n}\right)$,

$$
P_{\left(n_{p}, n_{n}\right)}=\prod_{\tau=p, n} \int_{0}^{2 \pi} \frac{d \varphi_{\tau}}{2 \pi} e^{i \varphi_{\tau}\left(N_{\tau}-n_{\tau}\right)}
$$

We can then handle $w^{(\mathrm{C})}$ and $\langle O\rangle_{\beta}^{(\mathrm{C})}$ in the grand-canonical ensembles,

$$
\begin{gathered}
w^{(\mathrm{C})}=\frac{e^{-\beta\left(H-\sum_{\tau} \mu_{\tau} n_{\tau}\right)}}{\operatorname{Tr}_{\mathrm{GC}}\left(P_{\left(n_{p}, n_{n}\right)} e^{-\beta\left(H-\sum_{\tau} \mu_{\tau} N_{\tau}\right)} P_{\left(n_{p}, n_{n}\right)}\right)}=\frac{e^{-\beta H}}{\operatorname{Tr}_{\mathrm{GC}}\left(P_{\left(n_{p}, n_{n}\right)} e^{-\beta H} P_{\left(n_{p}, n_{n}\right)}\right)}, \\
\langle O\rangle_{\beta}^{(\mathrm{C})}=\operatorname{Tr}_{\mathrm{GC}}\left(P_{\left(n_{p}, n_{n}\right)} O w^{(\mathrm{C})} P_{\left(n_{p}, n_{n}\right)}\right) .
\end{gathered}
$$

In practical calculations the integral in Eq. (11) is implemented by a finite sum of the $\varphi$ variables. Particularly when we work in a finite model space as below, the integral operator is identical to the operator represented by the discrete sum,

$$
\int_{0}^{2 \pi} \frac{d \varphi}{2 \pi} e^{i \varphi(N-n)}=\frac{1}{D+1} \sum_{m=0}^{D} e^{i \varphi_{m}(N-n)} ; \quad \varphi_{m}=\frac{2 m \pi}{D+1},
$$

where $D$ is the number of the single-particle states in the model space.

In the FT-MF theories, $H_{0}$ is determined by variation as mentioned above. In the variation-beforeprojection (VBP) method, $H_{0}$ is determined by the variation of $\Omega_{\mathrm{MF}}^{(\mathrm{GC})}$ in the grand-canonical ensemble, and the projection is applied when computing physical quantities $\left(\langle O\rangle_{\beta}\right)$. It should be noted, however, the VBP method violates the thermodynamical consistency [12]. Although the variationafter-projection (VAP) is a more desirable method, in which $H_{0}$ should be determined by minimizing the free energy $F_{\mathrm{MF}}^{(\mathrm{C})}$ in the canonical ensemble, the VAP has not been well formulated for the following reasons. 
The nuclear Hamiltonian satisfies $\left[H, N_{\tau}\right]=0$, and therefore $\left[H, P_{\left(n_{p}, n_{n}\right)}\right]=0$. Suppose that this also holds for the FT-MF Hamiltonian $H_{0} ;\left[H_{0}, N_{\tau}\right]=0$. It is then straightforward to apply $P_{\left(n_{p}, n_{n}\right)}$ to the FT-MF theory, by defining the canonical density operator by

$$
w_{\mathrm{MF}}^{(\mathrm{C})}=\frac{e^{-\beta\left(H_{0}-\sum_{\tau} \mu_{\tau} n_{\tau}\right)}}{\operatorname{Tr}_{\mathrm{GC}}\left(P_{\left(n_{p}, n_{n}\right)} e^{-\beta\left(H_{0}-\sum_{\tau} \mu_{\tau} N_{\tau}\right)} P_{\left(n_{p}, n_{n}\right)}\right)}=\frac{e^{-\beta H_{0}}}{\operatorname{Tr}_{\mathrm{GC}}\left(P_{\left(n_{p}, n_{n}\right)} e^{-\beta H_{0}} P_{\left(n_{p}, n_{n}\right)}\right)} .
$$

The VAP is formulated by minimizing the free energy $F=E-T S$, with the entropy

$$
\begin{aligned}
S_{\mathrm{MF}}^{(\mathrm{C})} & =-\operatorname{Tr}_{\mathrm{GC}}\left(\left[P_{\left(n_{p}, n_{n}\right)} w_{\mathrm{MF}}^{(\mathrm{C})} P_{\left(n_{p}, n_{n}\right)}\right] \ln \left[P_{\left(n_{p}, n_{n}\right)} w_{\mathrm{MF}}^{(\mathrm{C})} P_{\left(n_{p}, n_{n}\right)}\right]\right) \\
& =\beta \frac{\operatorname{Tr}_{\mathrm{GC}}\left(P_{\left(n_{p}, n_{n}\right)} H_{0} e^{-\beta H_{0}} P_{\left(n_{p}, n_{n}\right)}\right)}{\operatorname{Tr}_{\mathrm{GC}}\left(P_{\left(n_{p}, n_{n}\right)} e^{-\beta H_{0}} P_{\left(n_{p}, n_{n}\right)}\right)}+\ln \operatorname{Tr}_{\mathrm{GC}}\left(P_{\left(n_{p}, n_{n}\right)} e^{-\beta H_{0}} P_{\left(n_{p}, n_{n}\right)}\right) .
\end{aligned}
$$

However, the FT-MF Hamiltonian does not satisfy $\left[H_{0}, N_{\tau}\right]=0$ when the pairing correlation exists. Without this commutability, it is prohibitively difficult to use $S_{\mathrm{MF}}^{(\mathrm{C})}$ [16], as the equality of the second line of Eq. (16) is lost. Whereas an approximate VAP method has been proposed by respecting the Pierls inequality [16], assuming an approximate entropy

$$
S_{\mathrm{MF}}^{\prime(\mathrm{C})}=\beta \frac{\operatorname{Tr}_{\mathrm{GC}}\left(P_{\left(n_{p}, n_{n}\right)} H_{0} e^{-\beta H_{0}} P_{\left(n_{p}, n_{n}\right)}\right)}{\operatorname{Tr}_{\mathrm{GC}}\left(P_{\left(n_{p}, n_{n}\right)} e^{-\beta H_{0}} P_{\left(n_{p}, n_{n}\right)}\right)}+\ln \operatorname{Tr}_{\mathrm{GC}}\left(P_{\left(n_{p}, n_{n}\right)} e^{-\beta H_{0}} P_{\left(n_{p}, n_{n}\right)}\right),
$$

it again violates the thermodynamical consistency, leading to negative entropy at $T \approx 0$.

Despite the problems mentioned above, the particle-number projection is still useful in investigating influence of the particle-number conservation qualitatively. As mentioned above, the sharp transitions in the FT-MF theories do not remain in actual nuclei, closely connected to restoration of the broken symmetry. To investigate behavior around the critical temperature, the quantum-number projection provides us with a indispensable tool. In the present study, we shall apply the approximate VAP, which we shall abbreviate as VAP', as well as the VBP methods, keeping in mind their defects and limitations.

\subsection{State density in the saddle-point approximation}

The state density $\rho$ is quite relevant to the thermodynamics. It can be represented by the inverse Laplace transform of the partition function. By applying the saddle-point approximation, $\rho$ is approximately expressed in terms of the thermodynamical quantities. From the canonical partition function we reach [3]

$$
\rho^{(\mathrm{C})} \simeq \frac{e^{S^{(\mathrm{C})}}}{\sqrt{2 \pi \beta^{-2} C^{(\mathrm{C})}}},
$$

while from the grand-canonical partition function

$$
\rho^{(\mathrm{GC})} \simeq \frac{e^{S^{(\mathrm{GC})}}}{\sqrt{(2 \pi)^{3}\left(\Delta N_{p}\right)^{2}\left(\Delta N_{n}\right)^{2} \beta^{-2} C^{(\mathrm{GC})}}},
$$

with $\left(\Delta N_{\tau}\right)^{2}=\left\langle\left(N_{\tau}-n_{\tau}\right)^{2}\right\rangle_{\beta}^{(\mathrm{GC})}$.

It is recently argued that there could be an additional contribution to the denominator of Eq. (19) in the FT-MF framework, due to the proton- and neutron-number violation [12], because of $H_{0}$ itself depends on $\mu_{\tau}$. In the present study, we do not take it into account for the sake of simplicity. 
We compute the heat capacity $C$ by numerical differentiation of $E(\beta)=\langle H\rangle_{\beta}$, either in the canonical or the grand-canonical ensemble. The entropy $S^{(\mathrm{GC})}$ is given by Eq. (9) in the usual FT-MF theories in the grand-canonical ensembles. In the SMMC, $S^{(\mathrm{C})}$ is calculated by integrating $E(\beta)$, using the relation of the thermodynamical consistency

$$
S^{(\mathrm{C})}=\beta E+\ln Z^{(\mathrm{C})} ; \quad \ln Z^{(\mathrm{C})}(\beta)=\ln Z^{(\mathrm{C})}(0)-\int_{0}^{\beta} d \beta^{\prime} E\left(\beta^{\prime}\right) .
$$

In the particle-number projected MF approaches, the incommutability of $H_{0}$ and $N_{\tau}$ causes a problem again. If $\left[H_{0}, N_{\tau}\right]=0$, Eq. (16) provides $S_{\mathrm{MF}}^{(\mathrm{C})}$. If $\left[H_{0}, N_{\tau}\right] \neq 0$, Eq. (20) loses its ground. Even in that case, we again apply $S_{\mathrm{MF}}^{\prime(\mathrm{C})}$ in Eq. (17) to $\rho_{\mathrm{MF}}^{(\mathrm{C})}$ as an approximation.

The state density will be presented as a function of the excitation energy $E_{x}$, which is obtained by $E_{x}=E(\beta)-E_{\text {g.s. }}$ with $E_{\text {g.s. }}=\lim _{\beta \rightarrow \infty} E(\beta)$.

\subsection{Model space and effective Hamiltonian}

In this study we take several rare-earth nuclei as examples. For them the SMMC state densities are in good agreement with the experimental data $[8,10,11]$. The model space in the SMMC calculations is the full $Z=50-82$ shell plus $1 f_{7 / 2}$ for protons, and the full $N=82-126$ shell plus $0 h_{11 / 2}, 1 g_{9 / 2}$ for neutrons. The Hamiltonian is comprised of the monopole pairing and the quadrupole, octupole, hexadecupole interactions, by which dominant collective features are well taken into account. See Ref. [10] for details of the Hamiltonian. This SMMC setup is a good starting point to investigate CEF.

In Ref. [10], the CEF was argued by comparing the state densities obtained in the FT-HFB framework to those obtained from the SMMC calculations. The model space and the Hamiltonian in those FT-HFB calculations is identical to those in the SMMC. For most nuclei the FT-HFB approaches gives three phase transitions; the proton and neutron pairing transitions which occur at $E_{x} \lesssim 5 \mathrm{MeV}$, and the deformed-to-spherical transition which takes place at $E_{x} \gtrsim 8 \mathrm{MeV}$. To the best of our knowledge, there are no FT-HFB plus particle-number projection computer codes available for the shell model Hamiltonian. For this reason, we introduce the following effective Hamiltonian, to which the particle-number projection is applicable on top of the FT-BCS:

$$
H^{\prime}=\sum_{k} \varepsilon_{k}^{\prime} a_{k}^{\dagger} a_{k}-\sum_{\tau=p, n} g_{\tau}^{\prime} P_{\tau}^{\dagger} P_{\tau} ; \quad P_{\tau}^{\dagger}=\sum_{k>0(\in \tau)} a_{k}^{\dagger} a_{\bar{k}}^{\dagger} .
$$

Here $\varepsilon_{k}^{\prime}$ is fixed by the HF results at $T=0$. Although this ignores energy-dependence of the nuclear shape, it is not significant at energies below the critical temperature of the shape transition. $g_{\tau}^{\prime}$ is adjusted so as to reproduce the FT-HFB state densities. In practice, it is enhanced from the pairing strength $g_{\tau}$ in the original shell model Hamiltonian as $g_{\tau}^{\prime}=c_{\tau} g_{\tau}$, with $c_{p}=1.4$ and $c_{n}=1.6$. The enhancement factor $c_{\tau}$ should partially represent contribution of the multipole interactions to the pairing. We implement the FT-BCS calculations with $H^{\prime}$, and investigate influence of the restoration of the particle-number conservation by applying the VBP or the $\mathrm{VAP}^{\prime}$ method.

\section{Collective enhancement factor for state densities}

It has been known that the nuclear level densities calculated from MF theories underestimate the experimental data. This originates from the collective excitations which is not well described within the MF theories. To correct the influence of the collective excitations, the collective enhancement factor (CEF) $K$ is customarily introduced [1],

$$
\rho\left(E_{x}\right)=K\left(E_{x}\right) \rho_{\mathrm{MF}}\left(E_{x}\right) .
$$



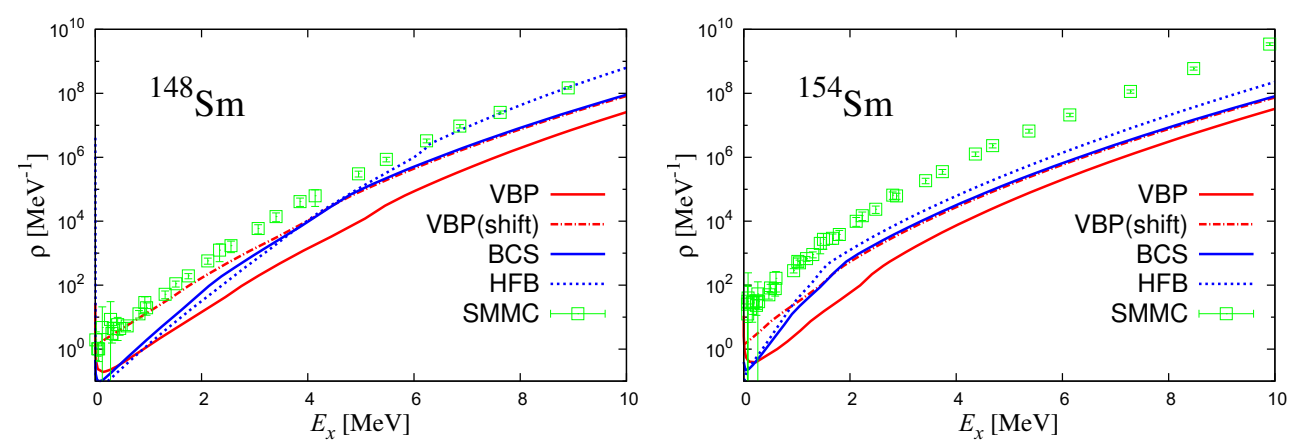

Figure 1. State densities $\rho\left(E_{x}\right)$ for ${ }^{148} \mathrm{Sm}$ and ${ }^{154} \mathrm{Sm}$. See text for details.

The MF density $\rho_{\mathrm{MF}}\left(E_{x}\right)$ is calculated by the FT-MF theory or by the combinatorial methods of counting the q.p. excitations [17]. The CEF contains effects of the collective vibration and the rotation, and may be factorized as

$$
K\left(E_{x}\right)=K_{\mathrm{vib}}\left(E_{x}\right) K_{\mathrm{rot}}\left(E_{x}\right) .
$$

For stable nuclei, experimental data on the state densities are obtained from direct counting of levels at low $E_{x}$ and from the neutron resonance around the neutron separation energy. The SMMC densities $\rho_{\mathrm{SMMC}}^{(\mathrm{C})}\left(E_{x}\right)$, which are available in the $\mathrm{Nd}-\mathrm{Sm}$ region $[10,11,18]$, are in good agreement with them. Since $\rho_{\mathrm{SMMC}}^{(\mathrm{C})}\left(E_{x}\right)$ gives state densities in wider energy range than the data, we shall use it as $\rho\left(E_{x}\right)$ in the lhs of Eq. (22).

It has been suggested in Ref. [10], by defining the CEF by $K\left(E_{x}\right)=\rho_{\mathrm{SMMC}}^{(\mathrm{C})}\left(E_{x}\right) / \rho_{\mathrm{HFB}}^{(\mathrm{GC})}\left(E_{x}\right)$, that the vibrational enhancement decays rapidly, in well correlation to the pairing phase transitions in the FT-HFB theory. Whereas it is difficult to separate the vibrational and rotational effects by comparing the MF density to the SMMC density, violation and restoration of the particle-number conservation should mainly be connected to the vibrational enhancement. At $E_{\text {pair }}<E_{x}<10 \mathrm{MeV}$ in the rare-earth nuclei, where $E_{\text {pair }}$ is the pairing energy, we have $K_{\text {vib }} \lesssim 3$ from an empirical formula [1].

\section{Numerical results of particle-number projection}

\subsection{State densities}

We take ${ }^{148} \mathrm{Sm}$ and ${ }^{154} \mathrm{Sm}$ as typical examples. ${ }^{148} \mathrm{Sm}$ is spherical with strong pairing correlation at $T=0$, while ${ }^{154} \mathrm{Sm}$ has quadrupole deformation with weak pairing [10]. In Fig. 1 several types of MF densities are compared with one another and with the SMMC densities. Among the MF densities, $\rho_{\mathrm{HFB}}^{(\mathrm{GC})}$ (blue dotted lines) is the FT-HFB results with the same Hamiltonian as in the SMMC. In the FTHFB calculations, the pairing transition occur at $E_{x} \approx 6 \mathrm{MeV}(1 \mathrm{MeV})$ for protons and $E_{x} \approx 4 \mathrm{MeV}$ $(2 \mathrm{MeV})$ for neutrons in ${ }^{148} \mathrm{Sm}\left({ }^{154} \mathrm{Sm}\right) . \rho_{\mathrm{BCS}}^{(\mathrm{GC})}$ (blue solid lines) is the results of the FT-BCS calculation with $H^{\prime}$ in Sec. 2.4. We observe $\rho_{\mathrm{BCS}}^{(\mathrm{GC})} \approx \rho_{\mathrm{HFB}}^{(\mathrm{GC})}$ to reasonable precision, at $E_{x} \lesssim 6 \mathrm{MeV}\left(E_{x} \lesssim 10 \mathrm{MeV}\right)$ for ${ }^{148} \mathrm{Sm}\left({ }^{154} \mathrm{Sm}\right)$. On this basis we use the FT-BCS results in place of the FT-HFB ones to investigate influence of the particle-number conservation. Red solid lines in Fig. 1 represent $\rho_{\mathrm{BCS}}^{(\mathrm{C})}$, which are obtained by applying the VBP when calculating $E(\beta)$ in the FT-BCS. We do not show the VAP' results because they are very close to the VBP results. 
In ${ }^{148} \mathrm{Sm}, \rho_{\mathrm{HFB}}^{(\mathrm{GC})}$ agrees well with $\rho_{\mathrm{SMMC}}^{(\mathrm{C})}$ above the pairing transitions, indicating no rotational enhancement in this spherical nucleus. In the deformed nucleus ${ }^{154} \mathrm{Sm}$, most of the HFB levels should correspond to the lowest states of the rotational bands, not including other members of each band. Thereby $\rho_{\mathrm{HFB}}^{(\mathrm{GC})}$ underestimates the state densities even above the pairing transitions, and the discrepancy stands for the rotational enhancement.

A notable effect of the particle-number projection is the suppression of the kink in the state densities at the pairing transition. This suggests much weaker energy-dependence of the CEF than reported in Ref. [10], if we properly take into account the conservation laws in the MF theories. We shall return to this point in the subsequent subsection.

If absolute values of $\rho_{\mathrm{BCS}}^{(\mathrm{C})}\left(E_{x}\right)$ is compared to $\rho_{\mathrm{BCS}}^{(\mathrm{GC})}\left(E_{x}\right)$, the particle-number conservation seems to reduce the state densities at all excitation energies under observation. This reduction might be attributed to elimination of the spurious states that have wrong particle numbers. In particular, the oneq.p. states give dominant contribution to the lowest-lying densities in the grand-canonical ensembles, but should not be present in practice. However, we should also keep in mind the negative entropy $\left(S_{\mathrm{MF}}^{\prime(\mathrm{C})} \approx-2\right.$ at $\left.T \approx 0\right)$, which may reduce the densities fictitiously. This problem obscures effect of the particle-number conservation on the absolute values of the state densities. It is noted as well that the MF densities have been shown to agree with the SMMC densities fairly well after appropriate corrections [12].

The particle-number projection lowers $E_{\text {g.s. }}$, by which the projected densities have shifted toward rhs in Fig. 1. To view influence of this energy shift, we also plot $\rho_{\mathrm{BCS}}^{(\mathrm{C})}$ by the VBP but as a function of $E_{x}^{\prime}=E_{\mathrm{BCS}}^{(\mathrm{C})}(\beta)-E_{\mathrm{BCS}}^{(\mathrm{GC})}(\infty)$ (red dot-dashed line), namely by taking $E_{\text {g.s. }}$ to be the value without the projection. Quite interestingly, we have $\rho_{\mathrm{BCS}}^{(\mathrm{C})}\left(E_{x}^{\prime}\right) \approx \rho_{\mathrm{BCS}}^{(\mathrm{GC})}\left(E_{x}\right)$ above the pairing transitions. Below the transitions, $\rho_{\mathrm{BCS}}^{(\mathrm{C})}\left(E_{x}^{\prime}\right)$ is greater than $\rho_{\mathrm{BCS}}^{(\mathrm{GC})}\left(E_{x}\right)$, in complete opposite to $\rho_{\mathrm{BCS}}^{(\mathrm{C})}\left(E_{x}\right) . \rho_{\mathrm{BCS}}^{(\mathrm{C})}\left(E_{x}^{\prime}\right)$ is close to $\rho_{\mathrm{SMMC}}^{(\mathrm{C})}$ below the transitions in ${ }^{148} \mathrm{Sm}$. Thus the particle-number projection gives improvement on the state densities, if the ground-state energy is properly shifted. These results seem consistent with those in Ref. [12].

\subsection{Collective enhancement factors}

The CEFs defined by $K\left(E_{x}\right)=\rho_{\mathrm{SMMC}}^{(\mathrm{C})}\left(E_{x}\right) / \rho_{\mathrm{MF}}\left(E_{x}\right)$ are shown in Fig. 2, for a number of even-even nuclei in the Nd-Sm region. To confirm effects of the particle-number conservation, we take $\rho_{\mathrm{BCS}}^{(\mathrm{GC})}\left(E_{x}\right)$ and $\rho_{\mathrm{BCS}}^{(\mathrm{C})}\left(E_{x}\right)$ (with the VBP) for the MF densities and compare the resultant CEFs.

As is expected from the state densities, the energy-dependence of the CEFs comes weaker after the particle-number projection. This indicates that the vibrational collectivity around the ground state remains up to high energy region. $\rho_{\mathrm{BCS}}^{(\mathrm{C})}\left(E_{x}\right)$ significantly lower than $\rho_{\mathrm{SMMC}}^{(\mathrm{C})}\left(E_{x}\right)$ gives high CEF. However, the problems for the absolute values of $\rho_{\mathrm{BCS}}^{(\mathrm{C})}\left(E_{x}\right)$ is inherited for the absolute values of $K\left(E_{x}\right)$, which prohibit us from making quantitative predictions based on the present calculations. The present results suggest that the CEF evaluated around the ground state will be important, not varying much up to relatively high energy.

The combinatorial methods based on the MF state at $T=0$ are sometimes applied to global description of the level densities. The combinatorial methods give a reasonable basis in evaluating the number of low-lying states. They could be suitable for estimate of the CEF around the ground state. Although they may miss energy-dependence of the nuclear mean fields as typified by the phase transitions, effects of the energy-dependence seem milder than predicted by the FT-MF theories. This may account for a part of the reasons why the combinatorial methods work moderately well. 


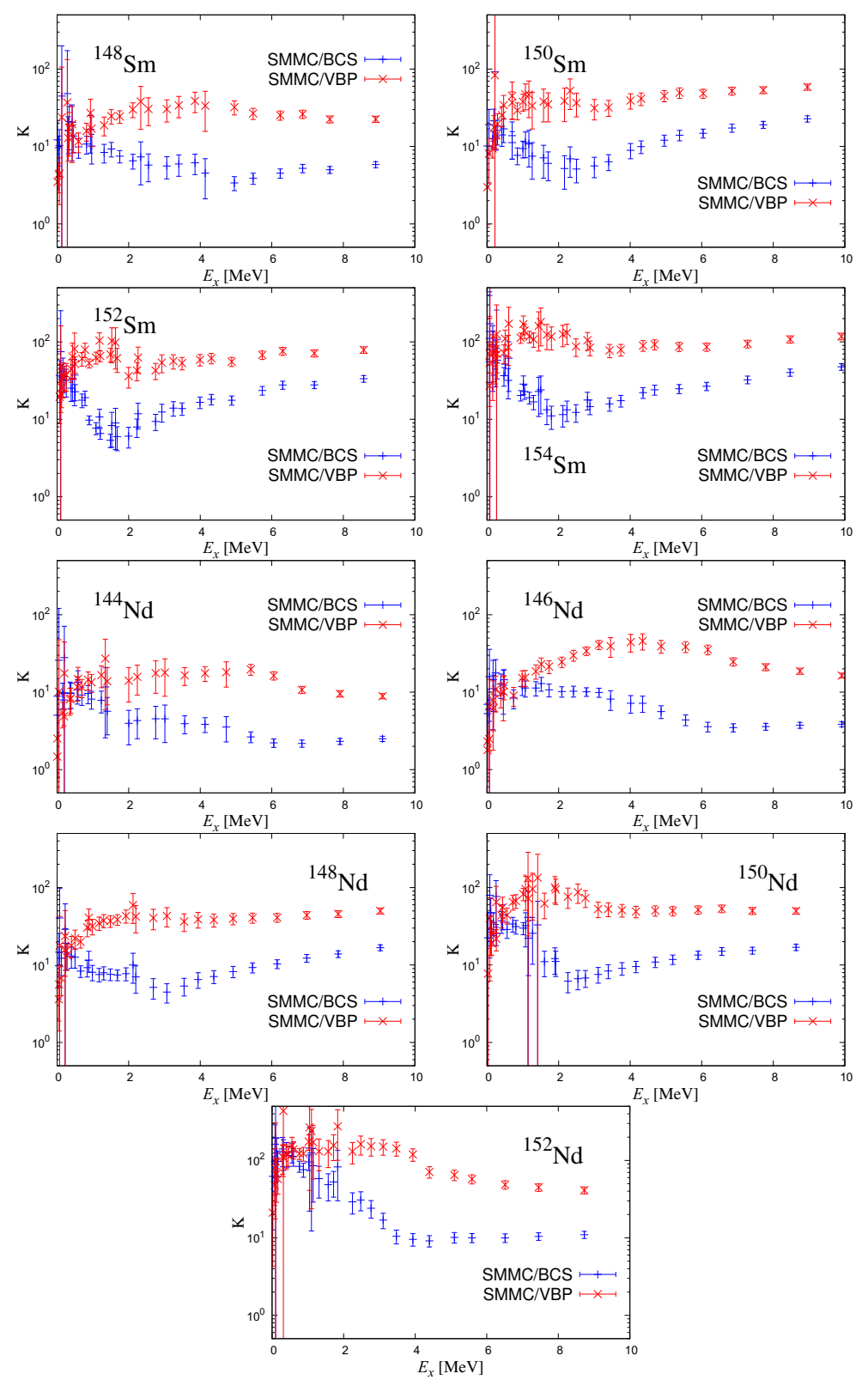

Figure 2. CEFs for Nd-Sm nuclei. Blue plus symbols and red crosses represent $\rho_{\mathrm{SMMC}}^{(\mathrm{C})}\left(E_{x}\right) / \rho_{\mathrm{BCS}}^{(\mathrm{GC})}\left(E_{x}\right)$ and $\rho_{\mathrm{SMMC}}^{(\mathrm{C})}\left(E_{x}\right) / \rho_{\mathrm{BCS}}^{(\mathrm{C})}\left(E_{x}\right)$ (with the VBP), respectively. 


\section{Summary and outlook}

We have investigated effects of the particle-number conservation on the nuclear state densities, particularly on their collective enhancement, by applying the projection to the finite-temperature (FT) mean-field (MF) theories. Although there remain problems in consistency in the projected FT-MF theories, qualitative effects can be observed from the projected results. It is found that the energydependence of the vibrational enhancement comes significantly weaker than the usual FT-MF results, almost washing out the decaying features of the collective enhancement factor (CEF) at the pairing transitions. This indicates that the vibrational collectivity around the ground state persists up to relatively high energy, in contrast to the unprojected FT-MF results.

Energy-dependence of the pairing correlation, which is manifested by the pairing transitions in the FT-MF theories, substantially affects the spin distribution of the level densities, as supported by the SMMC studies [19]. Even if the pairing transitions do not influence the state densities significantly after the particle-number conservation is restored, it is still of interest how they affect the spin distribution. Simultaneous implementation of the angular-momentum projection and the particle-number projection on the FT-MF theories [15] will enable us to investigate this point, though it is beyond scope of this paper and we leave it as a future subject.

The loss of the thermodynamical consistency has been a serious obstacle against quantitative arguments. It seems impractical to restore the consistency in the projected FT-MF theories. Still, there is a room of improvement. In the present study, we have adopted a simple effective Hamiltonian which is fitted to the FT-HFB state densities at low energy. This restricts the energy region of investigation. By applying the projection to the FT-HFB observables, it will be possible to extend the energy region and to investigate effects of the rotational symmetry in a similar manner, at least in a qualitative respect.

As mentioned above, the present results indicates significance of evaluating the CEF around the ground state. The combinatorial methods seem suitable for this purpose, and may help quantitative arguments, complementary to the FT-MF approaches, although they are unable to incorporate the energy-dependence of the pairing correlation appropriately.

\section{Acknowledgment}

One of the authors (H. N.) is grateful to G. F. Bertsch and Y. Alhassid for discussions. This work was supported by Grant-in-Aid for Scientific Research (C) No. 25400245 by the JSPS, Japan, and by the Turkish Science and Research Council (TÜBİTAK) grant No. ARDEB-1001-110R004 and ARDEB-1001-112T973.

\section{References}

[1] R. Capote et al., Nucl. Dat. Sheets 110, 3107 (2009).

[2] G. H. Lang, C. W. Johnson, S. E. Koonin and W. E. Ormand, Phys. Rev. C 48, 1518 (1993).

[3] H. Nakada and Y. Alhassid, Phys. Rev. Lett. 79, 2939 (1997).

[4] W. E. Ormand, Phys. Rev. C 56, R1678 (1997).

[5] H. Nakada and Y. Alhassid, Phys. Lett. B 436, 231 (1998).

[6] Y. Alhassid, S. Liu, and H. Nakada, Phys. Rev. Lett. 83, 4265 (1999).

[7] C. Özen, K. Langanke, G. Martinez-Pinedo and D. J. Dean, Phys. Rev. C 75, 064307 (2007).

[8] Y. Alhassid, L. Fang and H. Nakada, Phys. Rev. Lett. 101, 082501 (2008).

[9] H. Nakada and Y. Alhassid, Phys. Rev. C 78, 051304(R) (2008). 
[10] C. Özen, Y. Alhassid and H. Nakada, Phys. Rev. Lett. 110, 042502 (2013).

[11] C. Özen, Y. Alhassid and H. Nakada, Phys. Rev. C 91, 034329 (2015).

[12] Y. Alhassid, G. F. Bertsch, C. N. Gilbreth and H. Nakada, arXiv:1512.03773.

[13] C. Esebbag and J. L. Egido, Nucl. Phys. A 552, 205 (1993).

[14] R. Rossignoli and P. Ring, Ann. Phys. (N.Y.) 235, 350 (1994).

[15] K. Tanabe and H. Nakada, Phys. Rev. C 71, 024314 (2005).

[16] H. Nakada and K. Tanabe, Phys. Rev. C 74, 061301(R) (2006).

[17] A. J. Koning, S. Hilaire and S. Goriely, Nucl. Phys. A 810, 13 (2008).

[18] C. Özen, Y. Alhassid and H. Nakada, EPJ Web Conf. 69, 00011 (2014).

[19] Y. Alhassid, S. Liu, and H. Nakada, Phys. Rev. Lett. 99, 162504 (2007). 